\title{
The initial characterization of the iron environment in lipoxygenase by Mössbauer spectroscopy
}

\author{
William R. DUNHAM ${ }^{3}$, Richard T. CARROLL ${ }^{1}$, John F. THOMPSON ${ }^{2}$, Richard H. SANDS ${ }^{3}$ and Max O. FUNK, Jr ${ }^{1}$ \\ 1 Department of Chemistry, University of Toledo, Toledo, USA \\ 2 United States Plant Soil and Nutrition Laboratory, Agricultural Rescarch Service, United States Department of Agriculture, Ithaca, USA \\ 3 Biophysics Research Division, Institute for Science and Technology, University of Michigan, Ann Arbor, USA
}

(Received August 7, 1989) - EJB 890989

\begin{abstract}
The incorporation of ${ }^{57} \mathrm{Fe}$ into two lipoxygenase isoenzymes from soybeans has been achieved making possible the first observations of the iron environment in these proteins using Mössbauer spectroscopy. Immature soybean seeds were grown in tissue culture medium supplied with ${ }^{57} \mathrm{Fe}$. The iron in the active lipoxygenases that were isolated from the cultured seeds was readily detected in Mössbauer measurements. It was unequivocally demonstrated that the native enzyme contains high-spin Fe(II). Based on the sign of the electric field gradient, the most likely ligand sphere for the iron in native lipoxygenase consists of oxygen and nitrogen ligands in a roughly octahedral field of symmetry. It was possible to detect Mössbauer signals in highly concentrated samples of native lipoxygenases containing ${ }^{57} \mathrm{Fe}$ at natural abundance. The spectra obtained for enriched and natural abundance native enzyme had the same high-spin Fe(II) Mössbauer parameters. This confirmed that the environment of the iron in enzymes isolated from cultured seeds and dry soybeans were the same. The Mössbauer spectra $(4.2-250 \mathrm{~K})$ for samples of both isoenzymes after oxidation of the iron in native enzyme by the product of lipoxygenase catalysis were extremely broad $(20 \mathrm{~mm} / \mathrm{s})$ with no obvious narrow resonance lines. This was the result of the existence of paramagnetically broadened spectra for such samples even at relatively high temperature as evidenced by the appropriate EPR signal. A small molecule containing an iron site sharing many of these Mössbauer and electron paramagnetic resonance properties with lipoxygenase was identified: $\mathrm{Fe}(\mathrm{II}) /(\mathrm{III}) \cdot$ diethylenetriaminepentaacetic acid.
\end{abstract}

Lipoxygenases are responsible for an important fraction of polyunsaturated fatty acid metabolism in both plants and animals. The inaugural step in the conversion of arachidonic acid into 5-hydroxyicosatetraenoic acid and the leukotrienes in the mammalian circulatory system is catalyzed by 5 lipoxygenase [1]. Lipoxygenase-derived arachidonic acid metabolites have potent, medically relevant physiological activities in the immune response [2]. The biological purpose for lipoxygenase catalysis in plants is not so clear, but evidence for biosynthetic roles in the generation of growth-regulatory substances and pest-resistance compounds has been presented $[3,4]$. While the lipoxygenase found in soybeans has been known for some time [5], the details of the structure and mechanism of action of the enzyme are still in the process of being elucidated. The primary structures for the lipoxygenase 1 from soybeans [6] and the 5-lipoxygenase from leukocytes $[7,8]$ have been published recently. The degree of similarity among the sequences indicates that there may be common aspects of the structure which are presumably related to the catalytic mechanism.

All of the lipoxygenases available in sufficient quantity to be characterized have been found to contain $1 \mathrm{~mol} \mathrm{Fe} / \mathrm{mol}$ enzyme $[9,10]$. In lipoxygenase 1 the iron is necessary for catalysis and is thought to play an integral part in the mechanism of action [11]. It is thought that lipoxygenase 1, as it is isolated from soybeans, contains high-spin Fe(II). This was

Correspondence to M. O. Funk, Jr., Department of Chemistry, University of Toledo, 2801 West Bancroft Street, Toledo, OH 43606 , USA

Abbreviations. DTPA, diethylenetriaminepentaacetic acid; PQQ, pyrroloquinoline quinone; EFG, electric field gradient.

Enzyme. Lipoxygenase (EC 1.13.11.12). demonstrated by both classical magnetic susceptibility measurements [12] and paramagnetic susceptibility in NMR [13]. The native enzyme is EPR silent which is also a characteristic of high-spin Fe(II) [14]. Recent variable-temperature magnetic $\mathrm{CD}$ measurements were also consistent with the presence of $\mathrm{Fe}(\mathrm{II})$ in the native enzyme [15]. In comparison with the magnetic CD properties of iron complexes of known structure, it was suggested that the iron in native lipoxygenase 1 is sixcoordinate. The iron atom in native lipoxygenase has also recently been studied by extended X-ray absorption spectroscopy [16]. The observations were interpreted as being consistent with six nitrogen and/or oxygen ligands in the first coordination sphere. The recent detection of the phenylhydrazone of pyrroloquinoline quinone (PQQ) in proteolytic digests of lipoxygenase 1 treated with phenylhydrazine has raised the possibility that PQQ is in some way involved with iron binding [17].

The combination of lipoxygenase 1 with an equivalent amount of its product hydroperoxide results in significant changes in the EPR and ultraviolet/visible spectroscopic properties of the enzyme. A distinctive shift in absorbance to longer wavelength, which is characterized by a shoulder around $330 \mathrm{~nm}$, is observed in the spectrum [18]. This change gives the oxidized enzyme its characteristic yellow color in sufficiently concentrated samples. The reaction with product is also accompanied by the appearance of a complex signal at g6 in the EPR spectrum of treated samples. This has been attributed to the oxidation of the iron atom in native lipoxygenase 1 to $\mathrm{Fe}(\mathrm{III})$ [19]. The oxidized species is thought to be the active form of the enzyme [20].

The only previously reported attempt at the direct observation of lipoxygenase-1 iron by Mössbauer spectroscopy 
failed [21]. The inherent difficulty in this experiment is a result of the small amount of iron present in the protein $(0.06 \%$ by mass) combined with the low natural abundance $(2 \%)$ of the Mössbauer active isotope, ${ }^{57} \mathrm{Fe}$. In this paper we report a successful approach for overcoming these problems and present the first Mössbauer spectroscopic characterization of lipoxygenase. The key element in this approach was the isotopic enrichment of the enzyme by biosynthetic means. All of the previously reported biosynthetic enrichments with ${ }^{57} \mathrm{Fe}$ for Mössbauer spectroscopy have been performed with microbial proteins because the organisms can be conveniently grown in medium containing the isotope. Here we demonstrate for the first time that ${ }^{57} \mathrm{Fe}$ incorporation can be effectively achieved in a plant tissue grown in culture.

\section{EXPERIMENTAL PROCEDURES}

\section{Culture of immature seeds}

Immature seeds $(50-100 \mathrm{mg})$ were grown in culture as described previously [22]. Iron was supplied to the medium in the form of the diethylenetriaminepentaacetic acid (DTPA) chelate. Powdered iron $\left(94.45 \%{ }^{57} \mathrm{Fe}\right.$, New England Nuclear) was dissolved with warming in $6 \mathrm{M} \mathrm{HCl}$ and lyophilized to dryness. Separately, sodium bicarbonate $(843.2 \mathrm{mg})$ was gradually added to a solution of DTPA $(818.2 \mathrm{mg})$ in water $(75 \mathrm{ml})$. The chelate solution was diluted to a final volume of $100 \mathrm{ml}$. The dry $\mathrm{FeCl}_{3}$ was combined with $45.5 \mathrm{ml}$ chelate solution and warmed $\left(50^{\circ} \mathrm{C}, 30 \mathrm{~min}\right)$ to ensure complex formation. The clear, yellow orange solution (final concentration, $50 \mu \mathrm{M}$ ) was used as the stock for dispensing iron into the tissue culture medium.

\section{Enzyme isolation}

Cultured immature seeds $(60-100 \mathrm{~g})$ were frozen in liquid nitrogen and kept at $-80^{\circ} \mathrm{C}$ up to homogenization in icecold $25 \mathrm{mM}$ histidine, pH 5.7 (buffer A), $1 \mathrm{mM}$ phenylmethylsulfonyl fluoride followed by centrifugation $(16000 \times g$, $20 \mathrm{~min}, 4^{\circ} \mathrm{C}$ ). The supernatant was fractionated with solid ammonium sulfate $\left(30-60 \%\right.$ saturation, $\left.4^{\circ} \mathrm{C}\right)$. The precipitate was suspended in $16 \mathrm{ml}$ buffer $\mathrm{A}$ and dialyzed twice against 21 of this buffer at $4{ }^{\circ} \mathrm{C}$. The sample was centrifuged $\left(16000 \times \mathrm{g}, 20 \mathrm{~min}, 4^{\circ} \mathrm{C}\right)$ and the supernatant was applied to a chromatofocusing column (PBE 94, Pharmacia, $1.5 \mathrm{~cm}$ $\times 100 \mathrm{~cm}, 4^{\circ} \mathrm{C}$ ) that was equilibrated with buffer $\mathrm{A}$. The column was eluted with polybuffer 74 (Pharmacia, 1:12 di-

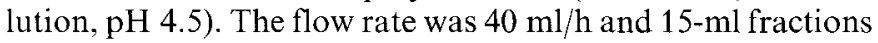
were collected. Active fractions were pooled and concentrated to $5 \mathrm{ml}$ with centrifugal concentrators (Amicon 30X Centriprep). The samples were dialyzed twice against 2-1 aliquots of $0.1 \mathrm{M}$ sodium phosphate, $0.1 \mathrm{M} \mathrm{NaCl}, 1 \mathrm{mM}$ EDTA, $\mathrm{pH}$ 7.0. Final concentration was carried out in centrifugal concentrators (Amicon $30 \mathrm{X}$ Centricon) by diafiltration at $4^{\circ} \mathrm{C}$ with $0.1 \mathrm{M}$ sodium phosphate, $0.1 \mathrm{M} \mathrm{NaCl}, \mathrm{pH} 7.0$, which had been passed through a column of Chelex 100. The final volume was $205 \mu \mathrm{l}$. The samples $(200 \mu \mathrm{l})$ were placed in Mössbauer cuvettes and frozen in liquid nitrogen. The remainder of the sample was used for the verification of purity.

\section{Mössbauer and EPR spectroscopy}

Mössbauer spectroscopic measurements were performed on an instrument that has been described in detail elsewhere [23]. Isomer-shift values are quoted relative to iron foil (NBS no. 1541) at standard temperature and pressure. The datareduction procedures include a deconvolution step to remove the effect of a finite source linewidth and a logarithmic conversion step to change the data from transmission to absorption as required by the Beer-Lambert law. The procedures increase the apparent signal-to-noise ratio so that high quality spectra can be obtained for natural abundance samples near $1 \mathrm{mM}$. The sample volume in all cases was $200 \mu \mathrm{l}$ with a $7.0-\mathrm{mm}$ sample diameter.

The above procedure results in spectra that are quantitative; they can be integrated to determine the amount of ${ }^{57} \mathrm{Fe}$ in the sample. However, the recoil-free fraction of the sample must be determined before one can quantify the data. This fraction [23] has a temperature dependence that can be matched theoretically by calculations based on the Debye model for solids. While we make no claims for its statistical mechanical significance, we report a parameter, $180 \mathrm{~K}$, as the sample Debye temperature that, when used in the abovementioned theory, removed the temperature dependence of the absorption mode data and allowed the bands to demonstrate a constant area irrespective of temperature.

The EPR spectra of the Mössbauer samples were taken on a Varian E-line Century series EPR spectrometer, which has been adapted so that its field modulation circuits will accommodate the V-4500 series wide-mouth cavity at $9 \mathrm{GHz}$. Sample positioning is important because the cavity operates in the TE-013 mode, and the short sample had to be located where both the field modulation and microwave $\mathrm{H}$-field were large.

\section{RESULTS}

To maximize the sensitivity of the Mössbauer measurements we employed isotopic substitution, efficient protein purification and signal-to-noise enhancing data-reduction procedures. Although the removal of iron from lipoxygenase by chelating agents has been reported, the reconstitution of the apoenzyme with added iron to give active lipoxygenase was not possible under a wide variety of experimental conditions [24]. The incorporation of iron isotopes into lipoxygenase biosynthetically was therefore investigated [25]. We have recently demonstrated the incorporation of ${ }^{59} \mathrm{Fe}$ into active lipoxygenase 1 by tissue culture of soybean seeds [26]. The lipoxygenases obtained from cultured seeds were found to be the same as those from mature seeds in specific activity and upon analysis via narrow-range isoelectric focusing, SDS/ gel electrophoresis, peptide mapping and chromatofocusing. The culture of seeds was repeated using ${ }^{57} \mathrm{Fe}(94.45 \%)$ supplied in the medium in the form of the DTPA chelate. Two lipoxygenase isoenzymes were isolated from the cultured seeds by extraction, ammonium sulfate fractionation and chromatofocusing. Samples of the isoenzymes were concentrated and dialyzed against buffer containing EDTA. It is known that lipoxygenase iron is not removed by EDTA nor is the enzyme inhibited by this treatment; therefore removal of traces of iron can be conveniently carried out in a dialysis step. The solutions were reduced in volume and washed by diafiltration in centrifugal concentrators using buffer not containing EDTA but which had been passed through a Chelex column. The purity of the isolated proteins was verified by SDS/PAGE.

Mössbauer samples were prepared for each isoenzyme from each of three independent preparations of cultured seeds. The resulting spectra for these samples are presented in Fig. 1. Each spectrum contains a clear doublet with isomer shift $(\delta /$ 

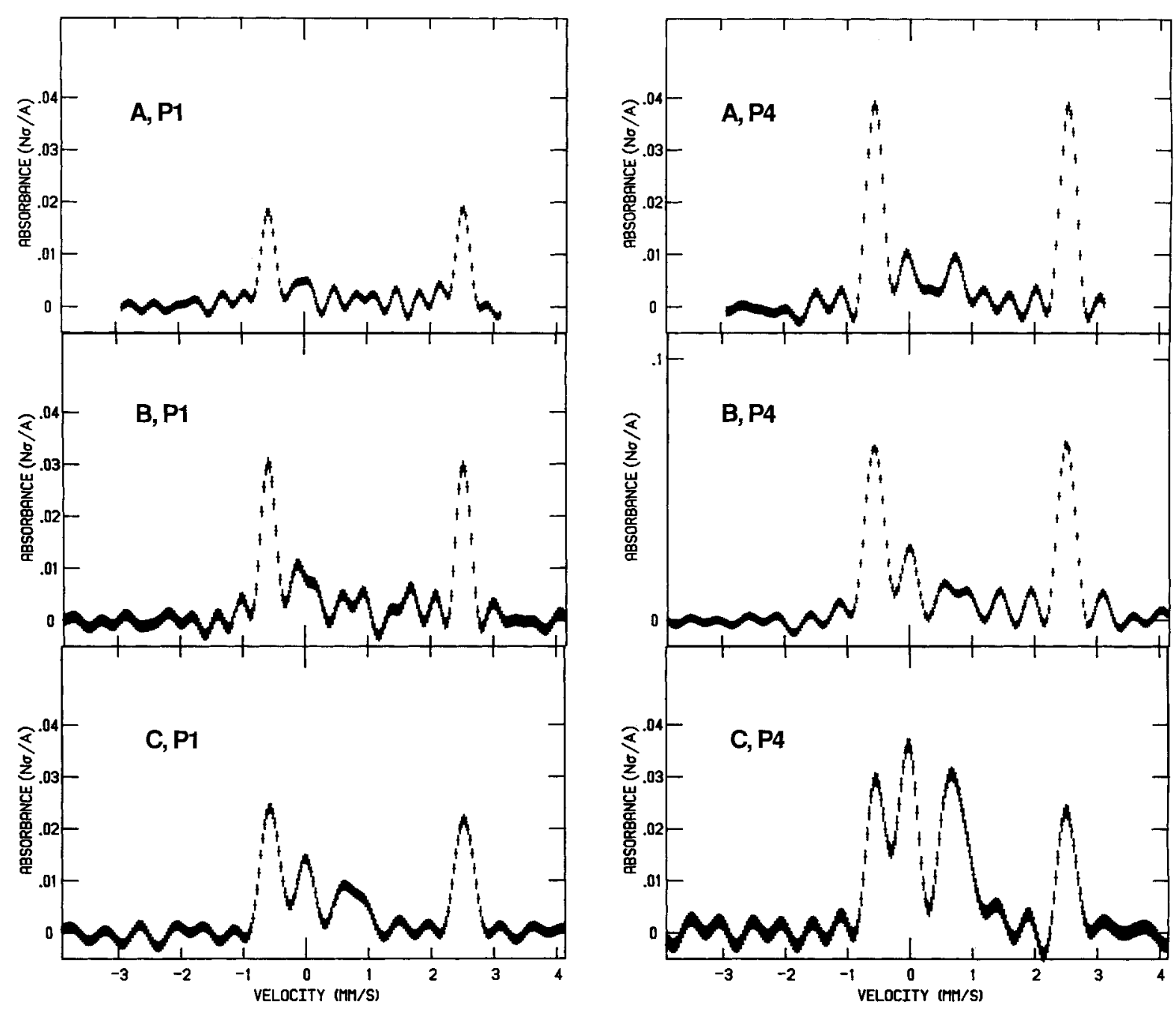

Fig. 1. Mössbauer spectra for three different preparations of two lipoxygenase isoenzymes, PI and P4, from seeds cultured in the presence of ${ }^{57}$ Fe. Conditions: $\mathrm{pH} 7.0$, phosphate, $0.1 \mathrm{M} \mathrm{NaCl}, 125$ 000. (A) P1, $0.235 \mathrm{mM} ; \mathrm{P} 4,0.482 \mathrm{mM}$. (B) P1, 0.80 mM; P4, $1.2 \mathrm{mM}$. (C) P1, 0.658 $\mathrm{mM} ; \mathrm{P} 4,0.438 \mathrm{mM}$. N, number of atoms; $\sigma,{ }^{57} \mathrm{Fe}$ resident absorption cross section; A, sample area

$\mathrm{Fe}=1.10 \mathrm{~mm} / \mathrm{s})$ and quadrupole splitting $\left(\Delta E_{\mathrm{q}}=3.08 \mathrm{~mm} /\right.$ s), parameters typical for high-spin $\mathrm{Fe}(\mathrm{II})$ [27]. This establishes unambiguously the oxidation number and spin state for the iron in native enzyme and provides confirmation for previous results from EPR, magnetic $C D$ and magnetic susceptibility with a direct observation of the metal atom. The spectra also contain a variable amount of a second doublet which is characteristic of $\mathrm{Fe}$ (III). The origin of this $\mathrm{Fe}$ (III) doublet is not presently clear. It is certainly not typical of the spectrum that is obtained for product-oxidized enzyme (vide infra). To be certain that the spectra for lipoxygenases obtained from cultured seeds were representative of the iron in the enzyme from mature seeds, spectra were obtained for each isoenzyme at natural abundance. This required not only a high concentration of the enzyme ( $>2 \mathrm{mM}$ ) but also an extended period of data collection. A clear doublet was obtained in each case with Mössbauer parameters identical to those obtained for the isoenzymes from cultured seeds (data not shown).

In Fig. 2 a comparison of the zero and high applied field spectra (superimposed by their fits) for lipoxygenase P4 is presented. The spectra were taken at $175 \mathrm{~K}$. The zero applied field spectrum (Fig. 2A) is fit with $\delta / \mathrm{Fe}$ equal to 1.08 $\pm 0.01 \mathrm{~mm} / \mathrm{s}, \Delta E_{\mathrm{q}}$ of $3.00 \pm 0.01 \mathrm{~mm} / \mathrm{s}$, and a line width (FWHM) of $0.15 \mathrm{~mm} / \mathrm{s}$. In the high-field spectrum, the fit has the same isomer shift and quadrupole splitting as for zero applied field, but with an asymmetry parameter $(\eta)$ equal to $0 \pm 0.1$ and the linewidth equal to $0.21 \mathrm{~mm} / \mathrm{s}$. The value of the applied field was $5 \mathrm{~T}$, but the value used in the fit was $3.8 \pm 0.1 \mathrm{~T}$. The large isomer shift and positive value of the largest component of the electric field gradient (EFG) tensor are characteristic of a high-spin $\mathrm{d}^{6}$ electronic configuration in a distorted octahedral field. A single $d$ electron generates a quadrupole splitting of around $3 \mathrm{~mm} / \mathrm{s}$. In a high-spin $\mathrm{d}^{6}$ configuration, the EFG tensor is dominated by the 'sixth' d electron, which resides in the d-orbital ground state. Thus a positive value for $\Delta E_{\mathrm{q}}$ eliminates the $\mathrm{d}_{\mathrm{z}}^{2}$ orbital as a possible ground state. Tetrahedral-ligand fields being weaker than octahedral fields also give rise to EFG tensors that are smaller and more temperature dependent than octahedral fields because the two states in the e set are more easily averaged than the three states of the $t$ set by thermal populations as the temperature increases. Therefore, a temperature-independent value of $+3 \mathrm{~mm} / \mathrm{s}$ for $\Delta E_{\mathrm{c}}$ indicates that the ground state of the orbital system is probably an isolated member of the $t$ set. A distorted octahedron provides this situation. Furthermore, all of the compounds with this combination of isomer shift and quadrupole splitting [27] also have a ligand sphere containing an octahedral array of oxygen and nitrogen atoms. 


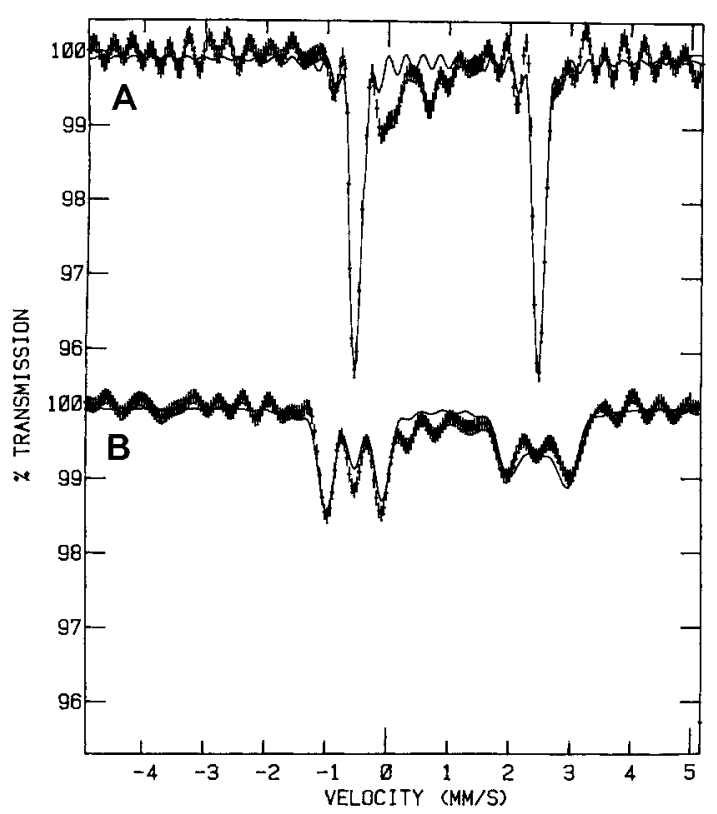

Fig. 2. Mössbauer spectra of the P4 lipoxygenase at $175 \mathrm{~K}$ at zero $(A)$ and 5-T (B) applied magnetic field (parallel to the $\gamma$-ray direction) superimposed by computer-simulated fits (solid lines). The data have been deconvolved for source lineshape and the Beer-Lambert law has been taken into account by the simulation program. The spectra are simulated from identical amounts of absorbing material. Slight mismatches result from a ferric impurity and the magnetic tensors in the Hamiltonian which were not taken into account (see text)

In the preceding paragraph it was mentioned that the magnitude of the best-fit value of the effective magnetic field was some $1.2 \mathrm{~T}$ below the actual field. From experiments performed on the $\mathrm{P} 1$ isoenzyme, we found that if one applies a $3-\mathrm{T}$ field at $125 \mathrm{~K}$, then the effective applied field is $0.8 \mathrm{~T}$ below the measured field. Lipoxygenase in the reduced state has its iron atom as a high-spin ferrous ion with $S=2$. At high temperatures and zero-applied magnetic field, the internal magnetic field that may accompany such a state is averaged to zero by spin-lattice relaxation. Therefore, at zero applied field the high-temperature spectra of native lipoxygenase show quadrupole pairs in their Mössbauer spectra. However, at high applied magnetic field the thermally averaged system gives rise to a net magnetic moment (Curie law), which produces a magnetic field at the nucleus via the magnetic hyperfine interaction that is opposite to the applied field. By calculating the size of the moment and the size of the internal field we can estimate the scalar portion of the magnetic hyperfine interaction as being near $9 \mathrm{~T} /$ electron, which compares well with the free ion value of approximately $11 \mathrm{~T} /$ electron [28]. The difference can be attributed to orbital reduction by the ligands. Our calculations show that the hightemperature moment is so insensitive to the value of the zerofield splitting parameters that we were unable to obtain any information on these numbers from the high temperature data although the lack of a perfect fit indicates that they are not zero.

The low-temperature, high-field data (Fig. 3D) were quite complex. At zero applied field and $4.2 \mathrm{~K}$ the spectrum of the reduced state was a quadrupole pair (not shown) with isomer shift of $0.96 \mathrm{~mm} / \mathrm{s}$ and quadrupole splitting of $3.13 \mathrm{~mm} / \mathrm{s}$. Thus the shapes of the $4.2 \mathrm{~K}$ spectra are very sensitive to the

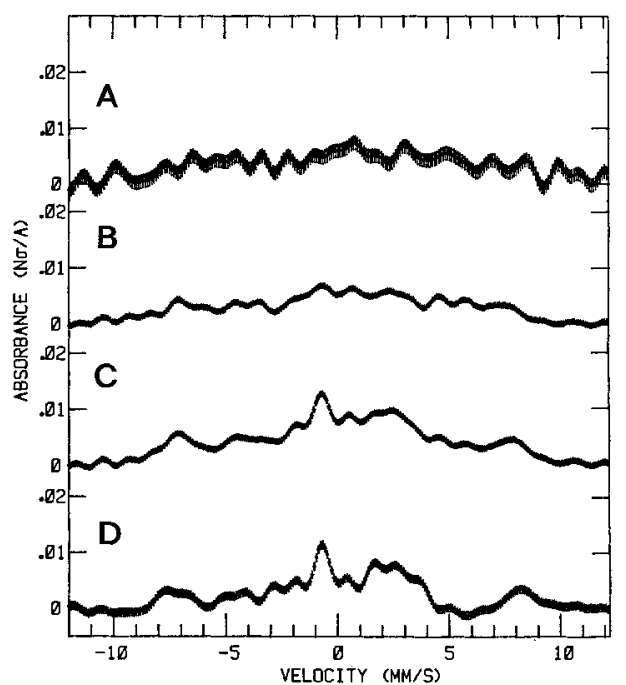

Fig. 3. Mössbauer spectra of two lipoxygenase isoenzymes after addition of equimolar 13-hydroperoxyoctadecadienoic acid, $\mathrm{pH}-7.0$ phosphate, $0.1 \mathrm{M} \mathrm{NaCl}$. (A) $125 \mathrm{~K}$, zero applied field spectrum of oxidized $0.64 \mathrm{mM}$ P1. (B) Difference spectrum $<$ (C) minus one-half (D) $>$ of oxidized $1.4 \mathrm{mM} \mathrm{P} 4$ isoenzyme taken at $4.2 \mathrm{~K}$ and 1 -T field applied parallel to the $\gamma$-ray direction. (C) $4.2 \mathrm{~K}, 1-\mathrm{T}$ spectrum of $1.4 \mathrm{mM} \mathrm{P} 4$ partially oxidized. (D) $4.2 \mathrm{~K}, 1$-T spectrum of $1.2 \mathrm{mM}$ reduced $\mathrm{P} 4$

applied magnetic field as expected for an integer-spin system. However these spectral shapes are also very sensitive to the values and orientations of the zero-field splitting and magnetic hyperfine tensors. We will leave this subject for a future article that compares these values with those of model compounds and includes a spectral synthesis program that diagonalizes the $5 \times 5$ electronic Hamiltonian as well as the nuclear Hamiltonian.

The Mössbauer spectra for samples obtained by combining the enriched form of each isoenzyme with a molar equivalent of the product of lipoxygenase-1 catalysis are presented in Fig. 3. A clear doublet absorption is not evident in either spectrum (Fig. 3A or 3B). Peak absorbances for the expected $\mathrm{Fe}(\mathrm{III})$ quadrupole doublet should have been in the range $0.03-0.05$ absorbance units for $\mathrm{P} 1$ and $\mathrm{P} 4$, respectively. The convex shape of the spectrum for P1 was obtained by setting the integrated absorbance equal to the expectation for the amount of iron present based on the known concentration of enzyme in the sample. However, the difference spectrum (Fig. 3B), for the product-oxidized P4 isoenzyme was the result of subtracting the spectrum of the reduced enzyme (Fig. 3D), scaled for concentration from Fig. 3C, obtained after a one-week run at $4.2 \mathrm{~K}$ and an applied magnetic field of $1 \mathrm{~T}$. This was carried out to account for the fact that approximately one-third of the sample gave rise to the $\mathrm{Fe}(\mathrm{II})$ signal. Either the enzyme was not completely oxidized by the product initially or partial reduction of the oxidized enzyme occurred during the concentration step. Both samples (Fig. 3C, D) quantified to approximately the same ${ }^{57} \mathrm{Fe}$ abundance ratio: $50 \%$. The Mössbauer spectrum is spread over $20 \mathrm{~mm} / \mathrm{s}$ due to the presence at the nucleus of magnetic hyperfine fields resulting from the $S=5 / 2$ Kramer's doublets. Because application of 5-T magnetic fields failed to sharpen the spectra, we are confident that the spectra shown in Fig. $3 \mathrm{~A}$ and $\mathrm{B}$ are the valid spectra for product-oxidized lipoxygenase. Below we present similar results for a compound for which the Fe(III) spectrum is more clearly seen. The important ob- 


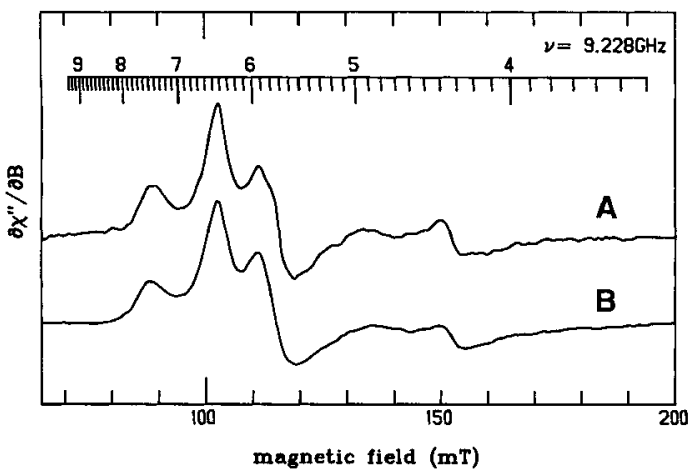

Fig. 4. EPR spectra of lipoxygenase PI after treatment with equimolar 13-hydroperoxyoctadecadienoic acid. (A) Mössbauer sample from Fig. 3. (B) Typical EPR spectrum for product-oxidized P1, $0.673 \mathrm{mM}$

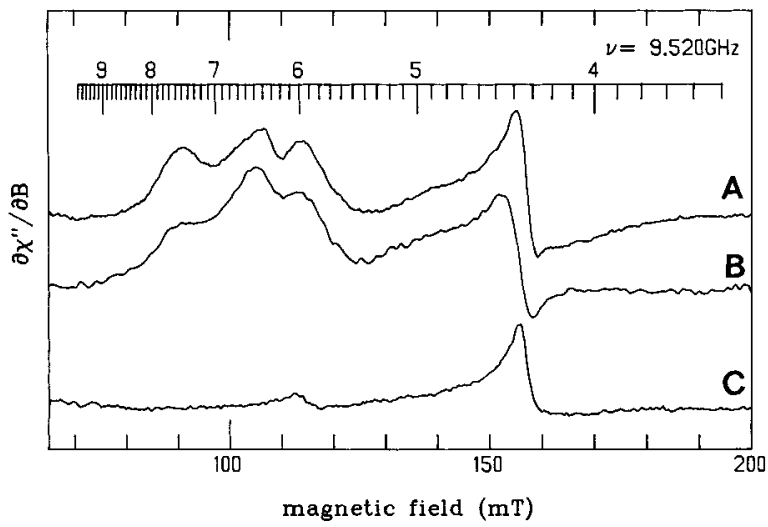

Fig. 5. EPR spectra of lipoxygenase P4 after treatment with equimolar 13-hydroperoxyoctadecadienoic acid. (A) Mössbauer sample from Fig. 3C. (B) Typical EPR spectrum for product-oxidized P4, 0.441 $\mathrm{mM}$. (C) Mössbauer sample from Fig. $1 \mathrm{C}$

servation for lipoxygenase is that for the product-oxidized enzyme, the resonances are spread over such a large range that no individual peaks are discernable even from ${ }^{57} \mathrm{Fe}$-enriched samples. To ensure that the samples contained the oxidized form of the enzymes, the EPR spectra for the Mössbauer samples were recorded. The EPR spectra for these samples, displayed in Figs $4 \mathrm{~A}$ and $5 \mathrm{~A}$, are the same as those for the corresponding product-oxidized isoenzymes from mature seeds, Figs 4B and 5B. A complex signal around g6 is clearly evident in each spectrum. By contrast, the EPR spectrum of the Mössbauer sample of the native enzyme characterized by the largest ferric doublet absorption (Fig. 1C, P4) does not contain the g6 signal (Fig. 5C). The absence of a clear doublet absorbance for Fe(III) in high-temperature Mössbauer spectroscopy is usually ascribed to the presence of a non-zero magnetic field at the nucleus due to the atomic electrons. The presence of a large magnetic field is attributable to the large moments of the three Kramer's doublets for the $S=5 / 2$ ion. The spin-relaxation process that allows for observation of the EPR signal does not in such instances average the electronic moment to zero as is common for Mössbauer spectra of highspin ferric compounds at high temperatures [29].

It should also be noted that running the Mössbauer spectra at $4.2 \mathrm{~K}$ and at applied fields of up to $5 \mathrm{~T}$ had no apparent effect on the spectra shown in Fig. 3. However, this is not surprising because the internal field of the atom is the reason
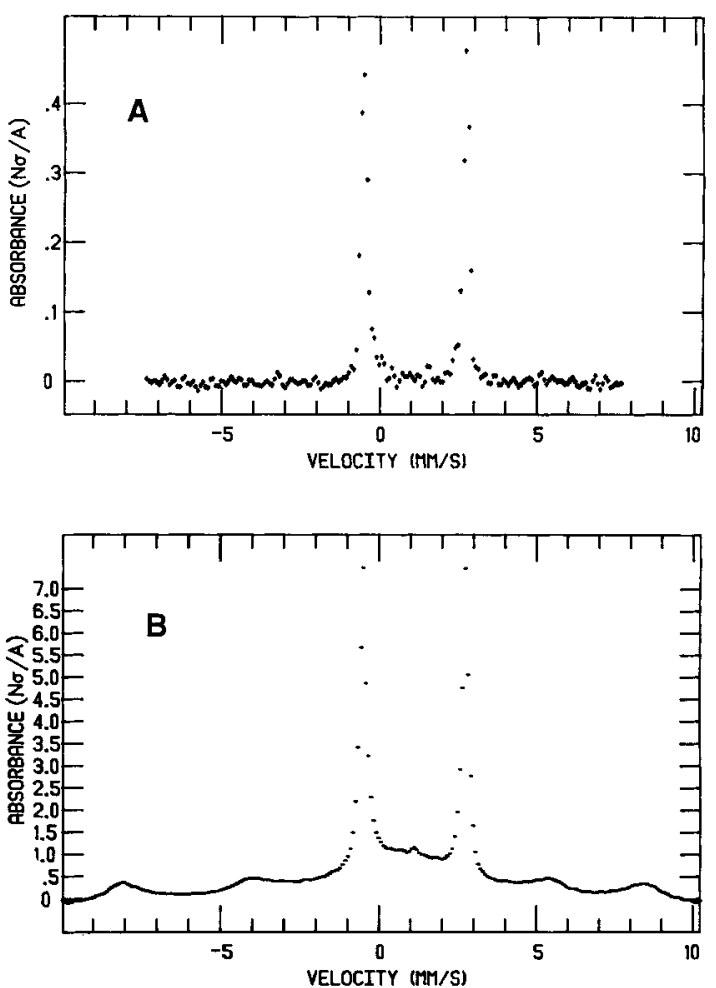

Fig. 6. Mössbauer spectra of Fe(II/III) - DTPA, $125 \mathrm{~K}$. (A) $125 \mathrm{mM}$ $\mathrm{Fe}(\mathrm{II}), \mathrm{pH}$ 7.0. (B) $125 \mathrm{mM} \mathrm{Fe}(\mathrm{II} / \mathrm{III})$, solution used to prepare (A) exposed to oxygen, $\mathrm{pH} 7.0$

for the pattern at high temperatures; one should not expect that it would become larger or more well defined at low temperatures when it is present at every temperature we tried $(4.2-250 \mathrm{~K})$. In addition, this absence of a quadrupole doublet is also a characteristic of the model compound $[\mathrm{Fe}(\mathrm{III}) \cdot \mathrm{DTPA}]$ described below. The enzyme and the model compound both display EPR signals at 0.15 -T over virtually the entire temperature range so that these spin systems must have a spin-lattice relaxation process available simultaneous to their absence of a quadrupole doublet; however, the spinlattice relaxation rate must be inadequate to average the ${ }^{57} \mathrm{Fe}$ magnetic hyperfine interaction. By performing saturation studies on the g6 region of the EPR signal from oxidized lipoxygenase at $125 \mathrm{~K}$, we are able to set a maximum on the spin-lattice-relaxation time of $30 \mathrm{~ns}$. Since the nuclear lifetime for the excited Mössbauer state is $100 \mathrm{~ns}$, it is possible for the spin relaxation to give rise to a broadened spectrum at all temperatures (i.e. one with no sharp lines).

As an illustration of a molecule with similar Mössbauer spectroscopic properties to the lipoxygenases, we present the preliminary results of an investigation of the complexes of iron with DTPA. The spectra obtained for Fe(II) - DTPA and Fe(III) - DTPA are presented in Fig. 6. The partially oxidized sample (Fig. 6B) was obtained by passing oxygen through the same solution used to prepare the sample for Fig. 6A. The parameters for the $\mathrm{Fe}(\mathrm{II})$ spectrum $\left(\delta / \mathrm{Fe}=1.24 \mathrm{~mm} / \mathrm{s}, \Delta E_{\mathrm{q}}=\right.$ $3.28 \mathrm{~mm} / \mathrm{s}$ ) are quite similar to those obtained for the native enzyme (Fig. 1). Further, the Fe(III) complex has an EPR signal that has resonances at 6,6 and 2 rather than the g4.3 signal from most high-spin ferric, weak-field ions. In Fig. 6 B some of the individual features from the broad resonance from this kind of high-spin ferric compound are apparent. In this 
spectrum it is possible to compare the strength of the Fe(II) lines which represent $40 \%$ of the integrated intensity with the weakness of the $\mathrm{Fe}$ (III) lines which represent the major component $(60 \%)$ of the sample. The details of our investigation of the EPR and Mössbauer spectroscopy of $\mathrm{Fe} \cdot \mathrm{DTPA}$ compounds will be reported elsewhere. The important point here is that these compounds mimic some of the spectroscopic properties of the lipoxygenases (isomer shift and quadrupole splitting in the reduced state as well as a wide spectrum over the $4.2-250 \mathrm{~K}$ temperature range in the oxidized state, plus an EPR signal at 6,6 and 2) and some of the chemical properties (resistance to oxidation by molecular oxygen).

\section{DISCUSSION}

The predominant absorption in the Mössbauer spectra for both isoenzymes of native soybean lipoxygenase was a quadrupole doublet described by parameters that are typical for high-spin $\mathrm{Fe}(\mathrm{II})$ [27]. The same spectrum was obtained for each isoenzyme whether isolated from mature soybeans or cultured immature seeds. This observation, along with our previous results, indicates that the lipoxygenases obtained from cultured immature seeds are equivalent to those that have been studied for some time from soybeans [26]. That is, the tissue-culture experiment does not introduce any detectable alteration in the structure or activity of the enzyme. The spectra for the two native isoenzymes have the same Mössbauer parameters indicating the similarity of the two iron sites. If the iron-binding sites of the isoenzymes are the same as these results appear to indicate, then differences in the active site residues other than the direct ligand amino acids will be found to be responsible for the differences in catalytic properties of the isoenzymes, e.g. $\mathrm{pH}$ optimum.

Comparisons of the observed Mössbauer parameters for the lipoxygenases with values obtained for other proteins reveal similarities with high spin $\mathrm{Fe}(\mathrm{II})$ in both heme and nonheme environments. The isomer shift of the iron in native lipoxygenase coincides with that for a number of hemeproteins [30]. The magnitude of the quadrupole splitting in most heme spectra is less than that found for lipoxygenases, although a value of $4.21 \mathrm{~mm} / \mathrm{s}$ has been found for the cytochrome $P 460$ center of hydroxylamine oxidoreductase [31]. Small molecule models for the high-spin Fe(II) environment in heme proteins include compounds with isomer shifts near $1 \mathrm{~mm} / \mathrm{s}$ and quadrupole splittings of $2.57 \mathrm{~mm} / \mathrm{s}$ and $3.67 \mathrm{~mm} / \mathrm{s} \mathrm{[32].} \mathrm{These}$ molecules contain, in addition to the porphyrin ring, one or two oxygen-containing ligands completing the coordination sphere.

The Mössbauer parameters for the Fe(II) component in the spectra of the lipoxygenases are also similar to those found in certain other non-heme-iron-containing dioxygenases. The value for the quadrupole splitting matches best with that obtained for dithionite-reduced protochatechuate 3,4-dioxygenases isolated from bacteria [33, 34]. The X-ray structure determination of the enzyme from Pseudomonas aeruginosa was reported recently [35]. The iron was found to be coordinated by two tyrosines, two histidines and a solvent molecule in an approximate trigonal bypyramid geometry. Related nonheme-iron dioxygenases tend to be characterized by iron/ tyrosinate interactions which give rise to distinctive ultraviolet/visible spectroscopic properties [36]. Histidine has also been implicated as a ligand in a study of the coordination sphere of catechol 1,2-dioxygenase [37]. Within this general class of enzymes, protochatechuate 4,5-dioxygenase has been found to be quite similar to the reaction center of the photosynthetic bacterium Rhodobacter sphaeroides [38, 39]. The recent report on the structure of the reaction center reveals the non-heme iron to be coordinated to four histidines and one bidentate glutamate residue [40].

Lipoxygenase is a non-heme protein and does not have the characteristic ultraviolet/visible spectroscopic properties of the iron-tyrosinate proteins. Therefore, we have sought model high-spin $\mathrm{Fe}(\mathrm{II})$ complexes containing simple amine- and carboxylic-acid ligand groups. As an example we present the spectrum of Fe(II) - DTPA. While the structure of this compound is not yet reported, by analogy to the similar complexes of Fe(III) with EDTA and DTPA, it is expected that six or seven oxygen and nitrogen ligands will be found to make up the coordination sphere [41]. The similarities between the Mössbauer parameters for native lipoxygenases and those for heme, non-heme, and model iron complexes along with results from other spectroscopic methods make it reasonable to propose a coordination sphere consisting of six oxygen and nitrogen ligand atoms in a roughly octahedral field of symmetry.

The Mössbauer spectra of the native lipoxygenases contain a variable contribution from a quadrupole doublet typical of Fe(III). This signal does not originate from the active, product-oxidized enzyme, which does not give a quadrupole doublet under these conditions. This interpretation was confirmed by the failure to detect the characteristic g6 signal in the EPR spectrum of the Mössbauer sample giving rise to the largest ferric doublet absorbance. Oxidation of the native enzyme by an equivalent amount of product apparently abolished the $\mathrm{Fe}(\mathrm{II})$ Mössbauer signal, but a quadrupole doublet from the expected Fe(III) species was not obtained. This phenomenon has been reported previously for the non-heme-iron dioxygenase, protochatechuate 3,4-dioxygenase [33]. The failure to observe quadrupole splitting in Mössbauer spectra is also characteristic of the Fe(III) in transferrin [42], ferrichrome A [29], and certain small-molecule complexes [43, 44]. We have found this behavior in our examination of the spectroscopy of Fe(III) - DTPA as well. Clearly, there are a number of examples of this phenomenon and the resulting spectra can be modeled using certain assumptions about electronic relaxation rates or internal magnetic fields [45]; however, a physical explanation for these observations is not yet apparent. It may be significant that the feature shared by complexes with these unusual Mössbauer properties is that they all have a large number of oxygen atom ligands in the coordination sphere.

This research was supported financially by the National Science Foundation and the National Institutes of Health. Max Funk is the recipient of a Research Career Development Award from the National Institutes of Health.

\section{REFERENCES}

1. Samuelsson, B., Dahlen, S.-E., Lindgren, J. A., Rouzer, C. A. \& Serhan, C. N. (1987) Science 237, 1171-1176.

2. Parker, C. W. (1987) Annu. Rev. Immunol. 5, 65-84.

3. Vick, B. A. \& Zimmerman, D. C. (1984) Plant Physiol. 75, 458461.

4. Preisig, C. L. \& Kuc, J. A. (1987) Plant Physiol. 84, 891-894.

5. Theorell, H., Holman, R. T. \& Akeson, A. (1947) Acta Chem. Scand. $1,571-576$.

6. Shibata, D., Steczko, J., Dixon, J. E., Hermodson, M., Yazdanparast, R. \& Axelrod, B. (1987) J. Biol. Chem. 262, $10080-10085$ 
7. Matsumoto, T. E., Funk, C. D., Radmark, O., Hoog, J., Jornvall, H. \& Samuelsson, B. (1988) Proc. Natl Acad. Sci. USA 85, 26 30 .

8. Dixon, R. A. F., Jones, R. E., Diehl, R. E., Bennett, C. D., Kargman, S. \& Rouzer, C. A. (1988) Proc. Natl Acad. Sci. USA $85,416-420$.

9. Chan, H. W. S. (1973) Biochim. Biophys. Acta 327, 32-35.

10. Rappoport, S. M., Schewe, T., Wiesne, R., Halangk, W., Ludwig, P., Janicke-Hone, M., Tunnert, C., Hiebsch, C. \& Klatt, D. (1979) Eur. J. Biochem. 96, 545-561.

11. Corey, E. J. \& Nagata, R. (1987) J. Am. Chem. Soc. 109, $8107-$ 8108.

12. Petersson, L., Slappendel, S. \& Vliegenthart, J. F. G. (1985) Biochim. Biophys. Acta 828, $81-85$.

13. Cheesbrough, T. M. \& Axelrod, B. (1983) Biochemistry 22, $3837-$ 3840.

14. Pistorius, E. K., Axelrod, B. \& Palmer, G. (1976) J. Biol. Chem $251,7144-7148$.

15. Whittaker, J. W. \& Solomon, E. I. (1986) J. Am. Chem. Soc. 108, $835-836$.

16. Navaratnam, S., Feiters, M. C., Al-Hakim, M., Allen, J. C., Veldink, G. A. \& Vliegenthart, J. F. G. (1988) Biochim. Biophys. Acta 956, 70-76.

17. van der Meer, R. A. \& Duine, J. A. (1988) FEBS Lett. 235, 194200.

18. Slappendel, S., Veldink, G. A., Vliegenthart, J. F. G., Aasa, R. \& Malmstrom, B. G. (1983) Biochim. Biophys. Acta 747, 32-36.

19. Slappendel, S., Veldink, G. A., Vliegenthart, J. F. G., Aasa, R. \& Malmstrom, B. G. (1981) Biochim. Biophys. Acta 667, 77-86.

20. DeGroot, J. J. M. C., Veldink, G. A., Vliegenthart, J. F. G., Boldingh, J., Wever, R. \& Van Gelder, B. F. (1975) Biochim. Biophys. Acta 377, $71-79$.

21. Slappendel, S., Veldink, G. A., Vliegenthart, J. F. G., Aasa, R. \& Malmstrom, B. G. (1980) Biochim. Biophys. Acta 642, 30-39.

22. Thompson, J. F., Madison, J. T. \& Muenster, A. E. (1977) Ann. Bot. $41,29-39$.

23. Dunham, W. R., Wu, C. T., Polichar, R. M., Sands, R. H. \& Harding, L. J. (1977) Nucl. Instrum. Methods 145, 537-553.

24. Pistorius, E. K. \& Axelrod, B. (1974) J. Biol. Chem. 249, $3183-$ 3186.
25. Skokut, T. A., Varner, J. E., Schaefer, J., Steiskal, E. O. \& McKay, R. A. (1982) Plant Physiol. 69, 308-313.

26. Funk, M. O., Carroll, R. T., Thompson, J. F. \& Dunham, W. R. (1986) Plant Physiol. 82,1139-1144.

27. Greenwood, N. N. \& Gibb, T. C. (1971) Mössbauer spectroscopy, pp. 112-147, Chapman and Hall, London.

28. Watson, R. E. \& Freeman, A. J. (1961) Phys. Rev. 123, $2027-$ 2047.

29. Wickman, H. H., Klein, M. P. \& Shirley, D. A. (1966) Phys. Rev. $152,347-357$.

30. Lang, G. (1970) Quart. Rev. Biophys. 3, 1-60.

31. Andersson, K. K., Kent, T. A., Lipscomb, J. D., Hooper, A. B. \& Munck, E. (1984) J. Biol. Chem. 259, 6833-6840.

32. Nasr, H., Fischer, J., Weiss, R., Bill, E. \& Trautwein, A. (1987) J. Am. Chem. Soc. 109, 2549-2550.

33. Que, L., Lipscomb, J. D., Zimmermann, R., Munck, E., OrmeJohnson, N. R. \& Orme-Johnson, W. H. (1976) Biochim. Biophys. Acta 452, 320-334.

34. Whittaker, J. W., Lipscomb, J. D., Kent, J. A. \& Munck, E. (1984) J. Biol. Chem. 259, 4466-4475.

35. Ohlendorf, D. H., Weber, P. C. \& Lipscomb, J. D. (1987) J. Mol. Biol. 195, 225-227.

36. Que, L. (1985) J. Chem. Educ. 62, $938-943$.

37. Que, L., Lauffer, R. B., Lynch, J. B., Murch, B. P. \& Purz, J. W (1987) J. Am. Chem. Soc. 109, 5381-5385.

38. Arciero, D. M., Lipscomb, J. D., Huynh, B. H., Kent, T. A. \& Munck, E. (1983) J. Biol. Chem. 258, $14981-14991$

39. Boso, B., Debrunner, P., Okamura, M. Y. \& Feher, G. (1981) Biochim. Biophys. Acta 638, 173-177.

40. Allen, J. P., Feher, G., Yeates, T. O., Komiya, H. \& Rees, D. C. (1987) Proc. Natl Acad. Sci. USA 84, 5730-5734.

41. Lind, M. D., Hamor, M. J., Hamor, T. A. \& Hoard, J. L. (1964) Inorg. Chem. 3, 34-43.

42. Tsang, C. P., Boyle, A. J. F. \& Morgan, E. H. (1973) Biochim. Biophys Acta 328, 84-94.

43. Knudsen, J. E. (1977) J. Phys. Chem. Solids 38, 883-896.

44. Bock, J. L. \& Lang, G. (1972) Biochim. Biophys. Acta 264, 245251.

45. Afanas'ev, A. M., Tsymbal, E. Y. \& Yakovleva, O. A. (1988) Hyperfine Interactions 39, 269-293. 